\title{
Performance Analysis of Wind-Induced Piezoelectric Vibration Bimorph Cantilever for Rotating Machinery
}

\author{
Gongbo Zhou, Houlian Wang, Zhencai Zhu, Linghua Huang, and Wei Li \\ School of Mechanical and Electrical Engineering, China University of Mining \& Technology, Xuzhou, Jiangsu 221116, China \\ Correspondence should be addressed to Gongbo Zhou; zhougongbo1985@163.com
}

Received 15 January 2015; Revised 7 April 2015; Accepted 7 April 2015

Academic Editor: Alicia Gonzalez-Buelga

Copyright (C) 2015 Gongbo Zhou et al. This is an open access article distributed under the Creative Commons Attribution License, which permits unrestricted use, distribution, and reproduction in any medium, provided the original work is properly cited.

\begin{abstract}
Harvesting the energy contained in the running environment of rotating machinery would be a good way to supplement energy to the wireless sensor. In this paper, we take piezoelectric bimorph cantilever beam with parallel connection mode as energy collector and analyze the factors which can influence the generation performance. First, a modal response theory model is built. Second, the static analysis, modal analysis, and piezoelectric harmonic response analysis of the wind-induced piezoelectric bimorph cantilever beam are given in detail. Finally, an experiment is also conducted. The results show that wind-induced piezoelectric bimorph cantilever beam has low resonant frequency and stable output under the first modal mode and can achieve the maximum output voltage under the resonant condition. The output voltage increases with the increase of the length and width of wind-induced piezoelectric bimorph cantilever beam, but the latter increasing amplitude is relatively smaller. In addition, the output voltage decreases with the increase of the thickness and the ratio of metal substrate to piezoelectric patches thickness. The experiment showed that the voltage amplitude generated by the piezoelectric bimorph cantilever beam can reach the value simulated in ANSYS, which is suitable for actual working conditions.
\end{abstract}

\section{Introduction}

With the continuous development of measurement and control technology of large mechanical and electrical equipment, the wireless sensors are widely used in the equipment monitoring system. Compared with the wired sensor, wireless sensors take the place of the wired communication cable with the help of wireless communication technology and substitute the portable power like battery for the wired power. As the wireless sensors can work without physical connection, it can be applied in the condition detection of middle-low-speed rotating machinery. In the process of practical application, the wireless communication module can avoid frequent maintenance, but the batteries need to be replaced frequently, especially in the detection of stress and vibration which has a large amount of data. Therefore, obtaining the energy contained in the running environment of rotating machinery to supplement energy to the battery and prolong the working life of the nodes is one of the problems that needs to be solved urgently.
Wind energy is a kind of typical energy in running environment of large mechanical and electrical system like mine hoist. For example, when rotary machine is working, there will be a certain relative velocity between rotary machine and air, resulting in the flowing air. So if the wind energy harvesting device is placed on the rotator, the wind energy can be collected in real time to power the wireless sensor nodes.

At present, the research on micro wind power generation technology is mainly divided into four categories: micro wind turbine generator, micro bellows generator, micro electromagnetic wind generator, and wind-induced piezoelectric energy harvesting device. Among these devices, the most mature and most commonly used one is the traditional wind power turbine technology, but under the condition of a small size, its power generation efficiency will decrease due to the increasing influence of the bearing friction loss and the decrease of the leaf area $[1,2]$; micro bellows generator has a higher power output when the wind speed is high $(>5.5 \mathrm{~m} / \mathrm{s})$, but the power output is very low when the wind 
speed is low $(<3.5 \mathrm{~m} / \mathrm{s})$; besides, it makes a lot of noise in the working process [3]; micro electromagnetic wind generator is reliable and the mechanical damping is small. But under the condition of low-speed wind, the efficiency of the generator will be influenced by the weight of the magnet [4]; windinduced piezoelectric energy harvesting device is cheap, easy to install, sensitive to the low-speed wind and also has a high efficiency of electromechanical conversion, small volume, light weight, compact structure, low magnetic permeability, and almost no heat loss [5]. Thus wind-induced piezoelectric energy harvesting technology is very suitable for the wireless sensors system.

The key point that influences the power generation efficiency of the wind-induced piezoelectric vibration energy harvesting device is the performance of energy collector. As the cantilever support mode can produce the biggest flexure coefficient and compliance coefficient [6], and the bent vibration frequency of the cantilever beam is usually lower than its longitudinal vibration frequency when it works as a shaft or its torsional vibration frequency when it works as an axle; besides, it can be motivated easily; most energy collectors choose the cantilever beam as their structure [7]. In addition, the wireless sensor nodes can work with a low voltage and the voltages generated by the two piezoelectric layers are the same; however, the current generated by piezoelectric bimorph is very low; thus parallel connection mode is often adopted to improve generator current in piezoelectric bimorph energy harvesting device. So this paper takes piezoelectric bimorph cantilever beam with parallel connection mode as energy collector and analyzes the factors that can influence the generation performance of the wind-induced piezoelectric vibration bimorph cantilever beam (WPB) by adopting the finite element method.

\section{Related Works}

Researchers have done a lot of works on piezoelectric vibration generator and made tremendous achievements. Priya et al. have manufactured a small generator [8] by using piezoelectric devices, which can provide up to the power of $50 \mathrm{~mW}$ for the wireless sensor nodes by increasing the number of piezoelectric bimorph in windmill. Tang and Zuo [9] proposed a spring-quality system generator model of double degree of freedom and calculated the maximum output power of the model; Arafa et al. [10] confirmed its feasibility by experiment. Challa et al. [11] and $\mathrm{Xu}$ et al. [12] established piezoelectric cantilever vibration generator model, respectively, from the perspective of adjusting the resonance frequency and broadening bandwidth. Sodano et al. [13] and DuToit et al. [14] built discrete mathematic model of piezoelectric vibration generator distributed-parameter system using the Rayleigh-Ritz discrete formula, but the methods simplify electromechanical coupling in the beam equation into viscous damping, and cannot consider resonance phenomena. Abd El-Mageed et al. [15] and Kiwata et al. [16] have conducted several experiments on energy harvester utilizing flow-induced vibration; however, the flow is liquid and not many literatures are focused on other flows like wind.

\section{Theory Model of Modal Response}

Piezoelectric bimorph cantilever belongs to Bernoulli-Euler beam as its thickness is far less than its length. Before building the theory model of modal response [17], this paper assumed a group of variables: the linear density of $\mathrm{WPB} \rho_{l}$, the bending stiffness of WPB $E_{m} I$ ( $E_{m}$ is the Young modulus of WPB and $I$ is product of inertia that cross section to neutral axle of WPB), the length from each point to the origin $x$, the base lateral displacement $g(t)$, and the base rotational displacement $h(t)$. The modal response satisfies the following ordinary differential equation:

$$
\frac{d^{2} q_{i}(t)}{d t^{2}}+2 \zeta_{i} \omega_{i} \frac{d q_{i}(t)}{d t}+\omega_{i}^{2} q_{i}(t)=N_{i}(t),
$$

where $\omega_{i}$ denotes the $i$ order undamped natural frequency of $\mathrm{WPB}, \zeta_{i}$ is the damping ratio of $\mathrm{WPB}$, and $N_{i}(t)$ is each order generalized force of PWB. And the damping ratio $\zeta_{i}$ satisfies the following equation:

$$
2 \zeta_{i} \omega_{i}=\frac{c_{s} \omega_{i}^{2}}{E_{m}}+\frac{c_{a}}{\rho_{l}}
$$

then, the damping ratio can be expressed as follows:

$$
\zeta_{i}=\zeta_{i}^{s}+\zeta_{i}^{a}=\frac{c_{s} \omega_{i}}{2 E_{m}}+\frac{c_{a}}{2 \rho_{l} \omega_{i}}
$$

where $c_{a}$ denotes the viscous air damping coefficient, $c_{s}$ is the strain rate damping coefficient, $\zeta_{i}^{s}$ is the strain rate damping ratio, and $\zeta_{i}^{a}$ is viscous air damping ratio. The strain rate damping coefficient $c_{s}$ and the viscous air damping coefficient $c_{a}$ can be expressed as

$$
\begin{aligned}
& c_{s}=\frac{2 \omega_{i} \omega_{j}}{\omega_{i}^{2}-\omega_{j}^{2}}\left(\frac{E_{m}}{\omega_{j}} \zeta_{i}-\frac{E_{m}}{\omega_{i}} \zeta_{j}\right), \\
& c_{a}=\frac{2 \omega_{i} \omega_{j}}{\omega_{i}^{2}-\omega_{j}^{2}}\left(\rho_{l} \omega_{i} \zeta_{j}-\rho_{l} \omega_{j} \zeta_{i}\right) .
\end{aligned}
$$

Each order generalized force of PWB composed of inertia force $N_{i}^{\rho}(t)$ and damping exciting force $N_{i}^{c}(t)$ can be expressed as

$$
N_{i}(t)=N_{i}^{\rho}(t)+N_{i}^{c}(t) .
$$

And the inertia force $N_{i}^{\rho}(t)$ and damping exciting force $N_{i}^{c}(t)$ can be expressed as

$$
\begin{aligned}
& N_{i}^{\rho}(t)=-\rho_{l}\left(\gamma_{i}^{w} \frac{d^{2} g(t)}{d t^{2}}+\gamma_{i}^{\theta} \frac{d^{2} h(t)}{d t^{2}}\right), \\
& N_{i}^{c}(t)=-c_{a}\left(\gamma_{i}^{w} \frac{d g(t)}{d t}+\gamma_{i}^{\theta} \frac{d h(t)}{d t}\right),
\end{aligned}
$$

where $\gamma_{i}^{w}=\int_{0}^{L} \phi_{i}(x) d x, \gamma_{i}^{\theta}=\int_{0}^{L} x \phi_{i}(x) d x$.

Finally, by using Duhamel integral, modal response can be expressed as

$$
q_{i}(t)=\frac{1}{\omega_{i d}} \int_{0}^{t} N_{i}(\tau) e^{-\zeta_{i} \omega_{i}(t-\tau)} \sin \omega_{i d}(t-\tau) d \tau,
$$

where $\omega_{i d}$ is damping natural vibration frequency. 


\section{Finite Element Modeling for WPB}

4.1. Parameters of the Model. The structure of WPB is shown in Figure 1. Piezoelectric ceramic PZT-5 is used as piezoelectric layer on both sides, and the intermediate is metal plate. Nickel alloy is selected as the metal layer and the mass block. The reference dimension of the WPB selected in this paper is shown as follows. The length, width, and thickness of the piezoelectric patches are $77 \times 42 \times 0.1 \mathrm{~mm}$. So does the metal layer.

Karman Vortex Street is a repeating pattern of swirling vortices caused by the unsteady separation of flow of a fluid around blunt bodies. So, when the wind hit WPB, Karman Vortex Street will occur. In other words, alternating shed vortex in the leeward wake of WPB will appear, causing the alternating vortex-excited force.

As is shown in Figure 2, the vortex-excited force can be divided into two forces, $F_{D}$ and $F_{L}$. The force component which is parallel with the flow is referred to as dragging force $F_{D}$, and the other one which is perpendicular to the flow is referred to as lifting force $F_{L}$. There are three main ways to calculate vortex-exited force. However, Morison equation [18] is usually selected in engineering practice. The expression of Morison equation is shown as follows:

$$
\begin{aligned}
F_{D} & =F_{M}+F_{C} \\
F_{M} & =\frac{\pi}{4} \rho C_{M} D^{2} \frac{\partial u}{\partial t} \\
F_{C} & =\frac{1}{2} \rho D C_{D} U^{2} \sin \left(\omega_{s}^{\prime} t\right) \\
F_{L} & =\frac{1}{2} \rho D C_{L} U^{2} \sin \left(\omega_{s} t\right) \\
\omega_{s}^{\prime} & =2 \omega_{s} .
\end{aligned}
$$

Among the equations, $\rho$ is the density of flow, $D$ is feature size of the object, and in this paper $D$ is $42 \mathrm{~mm}$, the width of PWB. $U$ is speed of the flow, $4 \mathrm{~m} / \mathrm{s}$ in this paper. $C_{D}$ is resistance coefficient which is only related to Reynolds number; $C_{D}=$ $f(\mathrm{Re}) . C_{M}$ is inertial coefficient, $C_{L}$ is lifting coefficient, $\omega_{s}$ is Strouhal frequency, and $\omega_{s}=2 \pi S_{t}(U / D)=89.714(14.3 \mathrm{~Hz})$ $\left(S_{t}\right.$ is the rectangular area which is 0.15 in this paper). When liquid is flowing around the object, the Reynolds number can be defined as

$$
\operatorname{Re}=\frac{D U}{v}
$$

where $v$ is dynamic viscosity. Under the condition of standard atmospheric pressure and 20 degrees centigrade, $v=1.513 \times$ $10^{-5} \mathrm{~m}^{2} / \mathrm{s}$.

When the angle of wind attack is $\pi / 2$ and Reynolds number is $10^{4}-10^{6}$, the resistance coefficient for two-dimensional plate that is perpendicular to the flow can be acquired from Table 1.

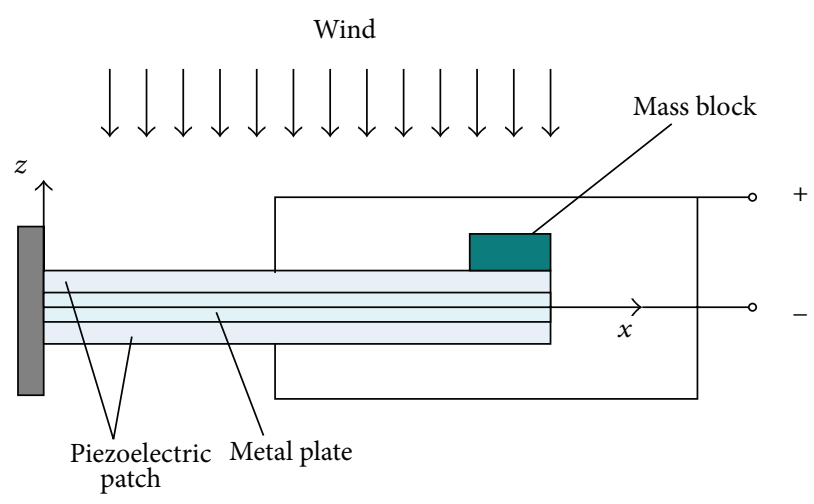

FIgURE 1: Structure diagram of WPB.

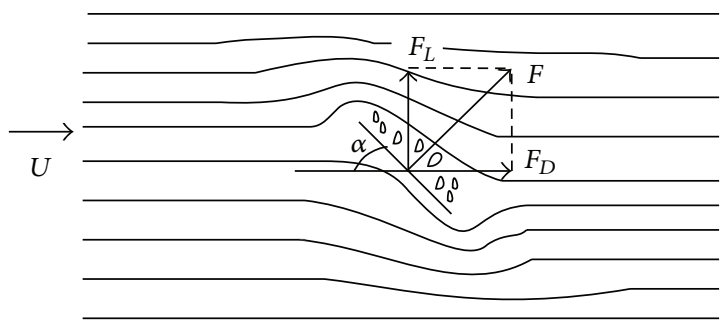

FIGURE 2: Vortex-excited force around blunt body structure.

The calculating result of lifting coefficient and resistance coefficient for the flow around plate under large attack angle can be expressed as follows:

$$
\begin{aligned}
& C_{D \alpha}=C_{D} \sin ^{2} \alpha \\
& C_{L \alpha}=\frac{C_{D}}{2} \sin 2 \alpha .
\end{aligned}
$$

As the angle of wind attack on WPB is $\pi / 2$, according to (10), $C_{L}=0$; thus $F_{L}=0$. Because the speed of wind is steady (in this paper, the WPB is deployed on the 2 JTB1.6 $\times 1.5-20$ mine hoist, and the linear velocity of WPB is $4 \mathrm{~m} / \mathrm{s}), \partial u / \partial t=$ 0 ; then, $F_{M}=(\pi / 4) \rho C_{M} D^{2}(\partial u / \partial t)=0$. Therefore, according to the Morison equation, the pressure intensity created by the fluctuating components of the average dragging force is

$$
F=\frac{1}{2} \rho C_{D} U^{2} \sin \left(2 \omega_{s} t\right)
$$

where $\rho=1.205$ under the condition of standard atmospheric pressure and 20 degrees centigrade. According to Table 1, $C_{D}=1.15$. Then $F=11.086 \sin (57.2 \times 3.1514 t)$.

4.2. Modeling and Meshing in ANSYS. The geometric model of WPB is shown in Figure 3(a). The finite element model corresponding to the solid geometry model can be acquired through mesh generation. We adopted the mapped meshing method as the mode of WPB is regular. The finite element model of WPB after meshing is shown in Figure 3(b).

Electrodes need to be defined to the piezoelectric cantilever and constrains need to be added to it after the finite element model was built. Both sides of the PZT layer are 
TABLE 1: Flat resistance coefficient.

\begin{tabular}{|c|c|c|c|c|}
\hline \multicolumn{2}{|l|}{ Object around the flow } & $b / D$ & Reynolds number Re & $C_{D}$ (angle of the attack is $\left.\pi / 2\right)$ \\
\hline & & 1 & & 1.12 \\
\hline & & 2 & & 1.15 \\
\hline & & 4 & $10^{4} \sim 10^{6}$ & 1.19 \\
\hline & & 10 & & 1.29 \\
\hline & D & 18 & & 1.40 \\
\hline$U \uparrow$ & $\downarrow$ & $\infty$ & & 2.01 \\
\hline
\end{tabular}

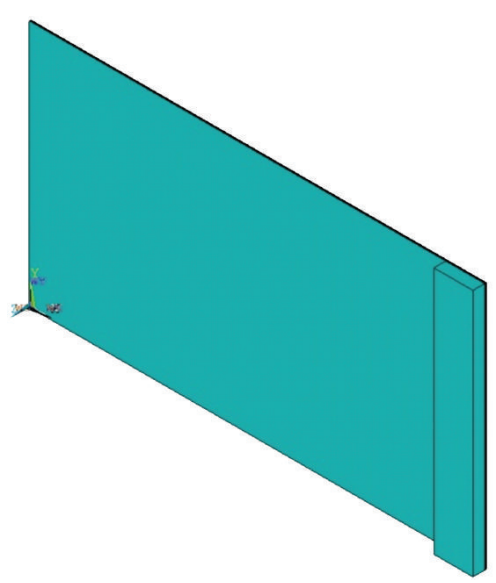

(a) Geometric model

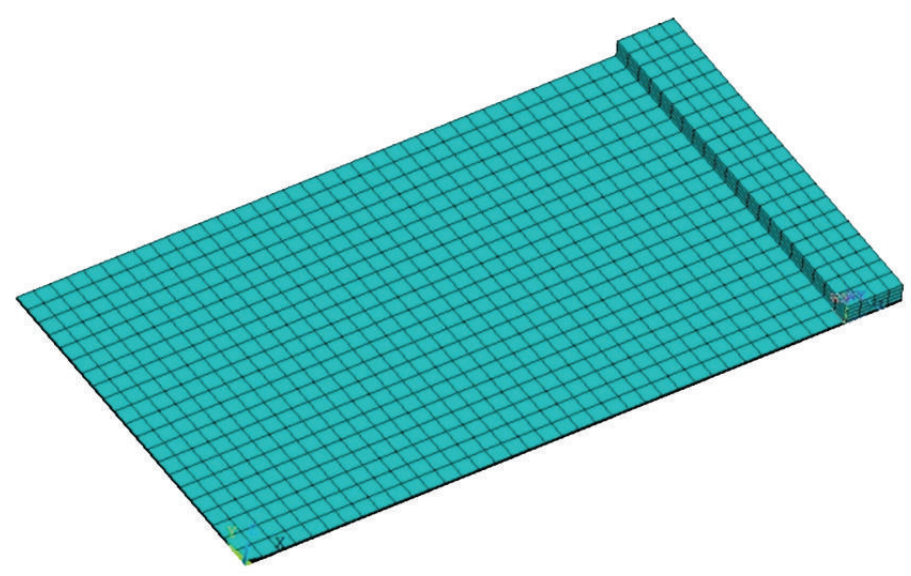

(b) After meshing

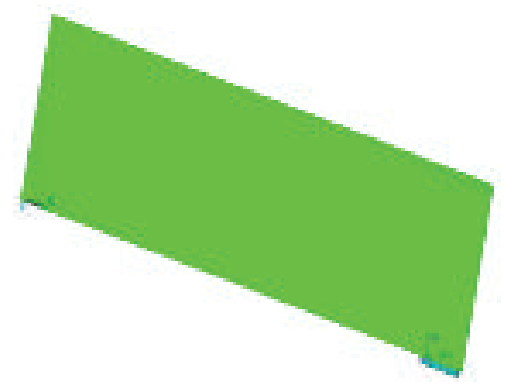

(c) After defining electrodes and constraints

Figure 3: The model of WPB.

defined as the electrodes, and the lower layer is earthed. The piezoelectric bimorph was set as parallel mode, and the freedom degree from one of the electrodes was set to 0 (that is being earthed). Because one end of piezoelectric cantilever beam model is fixed and the other one is free, three degrees of freedom (UX, UY, and UZ) on the surface that $x=0$ of the piezoelectric cantilever beam model are set to 0 . The result is shown in Figure 3(c).

4.3. Static Analysis. Statics analysis is mainly used to calculate the response of the structure under the action of fixed load, and it considers neither the influence of inertia and damping nor the change of load over time. Static pressure of $11.086 \mathrm{~N}$ (the amplitude of sine excitation on piezoelectric cantilever) was exerted on the surface of WPB and then solved the model. The Von-Mises stress nephogram is shown in Figure 4.

As shown in Figure 4, the maximum stress produced in the root of WPB is $2.25 \mathrm{MPa}$, which is less than the allowable stress of piezoelectric ceramic (60-100 MPa). Therefore, the exciting force exerted will not cause the WPB mechanical problems which result from the fatigue damage and assure the stable performance of the device.

4.4. Modal Analysis. Modal analysis is used to determine the natural frequency and vibration shape of WPB. The vortex-induced force results from the periodic shed vortex 


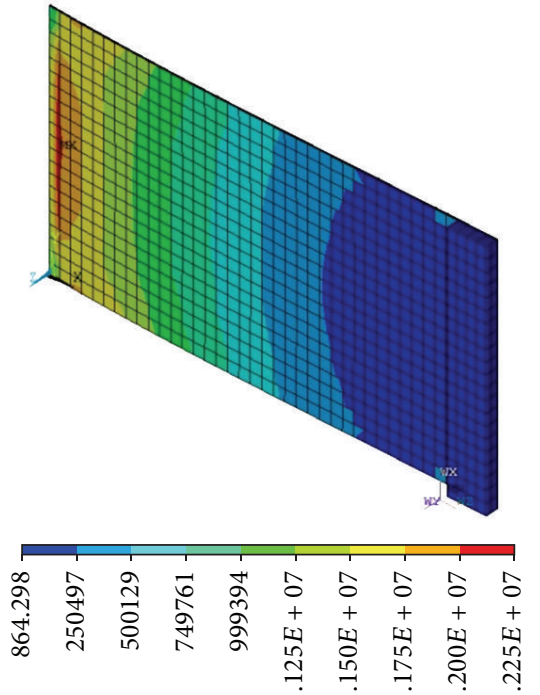

FIGURE 4: Von-Mises stress distribution map of WPB.

in the leeward wake for general structure can only cause smaller structural response. In order to make the WPB produce larger structure deformation, its natural frequency must come closer or be consistent to the vortex shedding frequency; only in this way will the vortex-induced resonance effect between WPB and air flow occur. In this paper, the vortex shedding frequency in the leeward wake of WPB under the basic size is $14.3 \mathrm{~Hz}$, so the size of mass at the free end can be adjusted to acquire the appropriate natural frequency of WPB. In other words, the size of mass at the free end needs to be adjusted to make the natural frequency up to $14.3 \mathrm{~Hz}$. At this point the dimensions of the mass at the free end are $L_{m}=6.6 \mathrm{~mm}, W_{m}=42 \mathrm{~mm}, H_{m}=2 \mathrm{~mm}$. The modal analysis results of the first six orders vibration modes of WPB are shown in Table 2.

As the vibration frequencies of the objects in the surrounding environment mostly range between $60 \mathrm{~Hz}$ and $200 \mathrm{~Hz}$ and the 5 and 6 orders natural frequencies of WPB are much greater than $200 \mathrm{~Hz}$, it is enough to analyse 1 to 4 orders vibration modes of WPB. The results are shown in Figure 5.

Figure 5 shows that the WPB under the first modal shape is only bobbing in the vertical direction without irregular distortion. At this point, the device has low resonant frequency and stable output, so the first modal shape is selected as the object for harmonic response analysis.

4.5. Harmonic Response Analysis. Harmonic response analysis is applied to determine the changing relationship between the output voltage and the frequency of WPB. The simulation results of harmonic response analysis are shown in Figure 6, where the target frequency range of harmonic response analysis is set to $5-26 \mathrm{~Hz}$, the number of the substeps is set to 70 , and the damping ratio is set to 0.02 . The figure shows that the output voltage achieves the maximum of $12.3 \mathrm{~V}$ when the WPB is under the resonant condition.
TABLE 2: The results of modal analysis.

\begin{tabular}{lcccccc}
\hline Vibration mode & 1 & 2 & 3 & 4 & 5 & 6 \\
Natural frequency & 14.30 & 62.80 & 123.94 & 296.26 & 376.06 & 662.71 \\
\hline
\end{tabular}

\section{Performances}

Wireless sensor nodes usually have smaller size, so we need to increase energy output as far as possible on the basis of minimizing the structure of WPB. Thus it is necessary for us to master the relationship between the power output and WPB size structure. By using ANSYS software, this section gives a detailed analysis of the modal and harmonic response of the WPB and the relationship among the output voltage, the structure size, and natural frequency of WPB.

The relationship among the output voltage, the natural frequency, and the length of WPB is shown in Figure 7. Under the condition of other parameters unchanged, the natural frequency decreases with the increase of the length of WPB and is inversely proportional to the square of the length approximately. So, the change of device length has a significant impact on natural frequency. Output voltage increases with the increase of the device length, and the relationship between them is close to linear.

Figure 8 shows the relationship among the output voltage, the natural frequency, and the width of WPB. Under the condition of other parameters unchanged, when the width of WPB is increased from $12 \mathrm{~mm}$ to $72 \mathrm{~mm}$, the resonance frequency of the device changes by less than $1 \mathrm{~Hz}$; it just means that the change of the width has little impact on the natural frequency of the device. The output voltage increases slowly with the increase of the device width, so we can infer that the change in width of WPB has little influence on the output voltage.

The relationship among the output voltage, the natural frequency, and the thickness of the WPB is shown in Figure 9. Under the condition of other parameters unchanged, the natural frequency increases with the increase of the thickness of WPB. Output voltage decreases with the increase of its thickness, and the relationship between the two is close to the inverse ratio.

The relationship between the output voltage of WPB and the ratio of the metal substrate to the piezoelectric patches thickness is shown in Figure 10. Under the condition of other parameters unchanged, the output voltage decreases with the increase of the ratio of metal substrate to piezoelectric patches thickness, and the extent of decrease is larger.

The relationship among the natural frequency, the output voltage, and the thickness of mass at the free end is shown in Figure 11. Under the condition of other parameters unchanged, the natural frequency decreases with the increase of the thickness of mass at the free end and is inversely proportional to the cubic root of the thickness of mass approximately. When the thickness of the mass is increased successively, the output voltage of the device changes by less than $0.1 \mathrm{~V}$, which means that the change of the mass thickness has little impact on the output voltage of the device. 


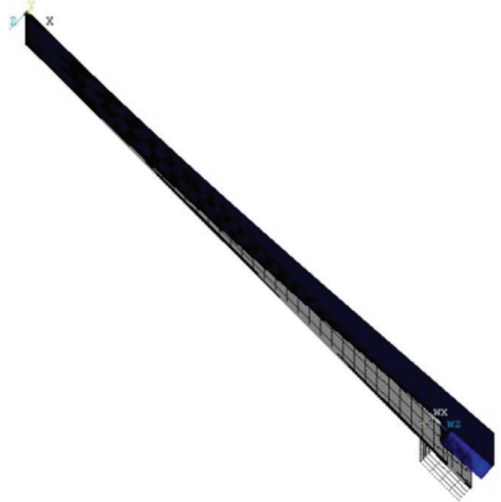

(a) First modal shape

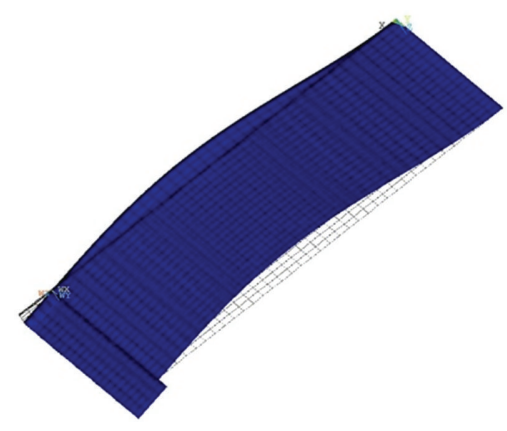

(c) Third modal shape

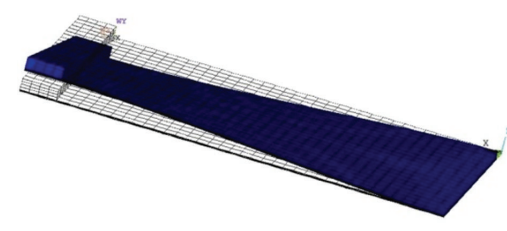

(b) Second modal shape

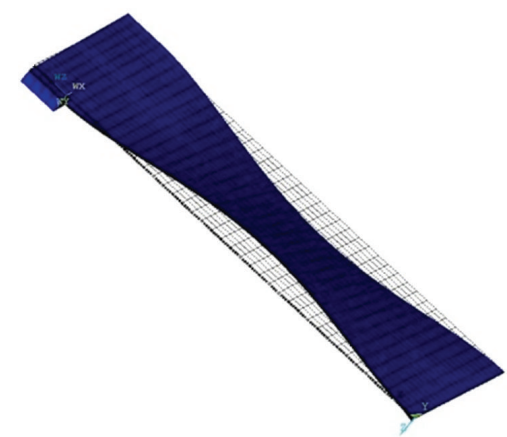

(d) Forth modal shape

Figure 5: Vibration shapes of the WPB.

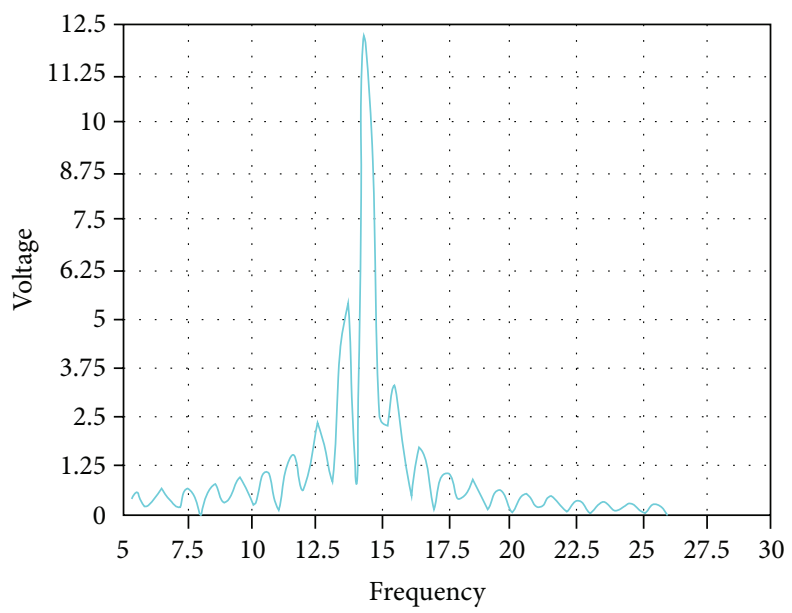

FIGURE 6: The harmonic response analysis of WPB.

\section{Experiment of WPB}

According to the parameters of the model in Section 4.1 of this paper, an experimental test system was built up. The experimental test system is shown in Figure 12. It is composed of simulation of hoist roller, piezoelectric bimorph cantilever beam, energy harvesting circuit, resistance divider, data acquisition and wireless transmitting module, and wireless receiving module. The wireless transmitting module sends the voltage generated by the piezoelectric bimorph cantilever beam and divided by resistance divider via nRF24L01 module and the wireless receiving module receives the data of voltage and updates it to host computer.

The output voltage amplitude under the condition that the wind velocity keeps raising in 300 seconds is shown in Figure 13. As is shown in Figure 13, the voltage value 


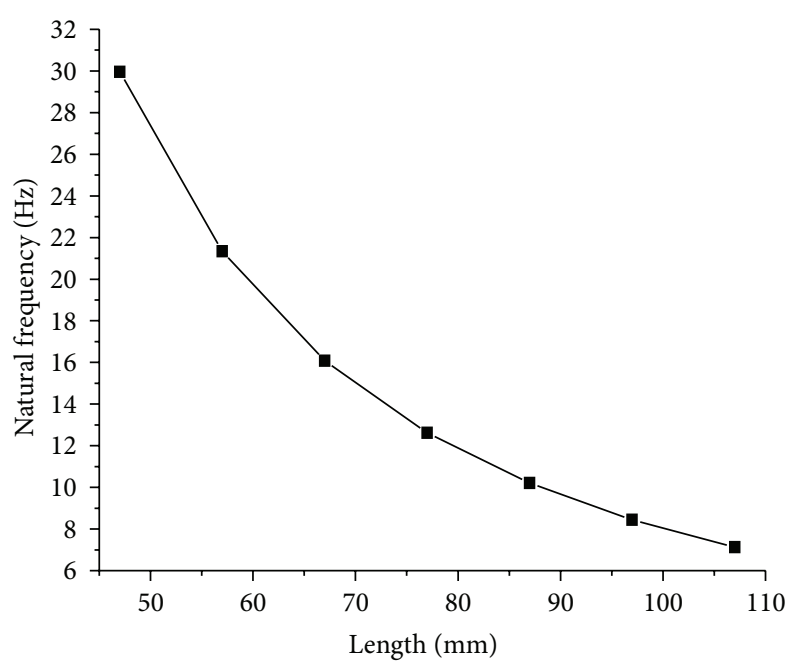

(a) Natural frequency

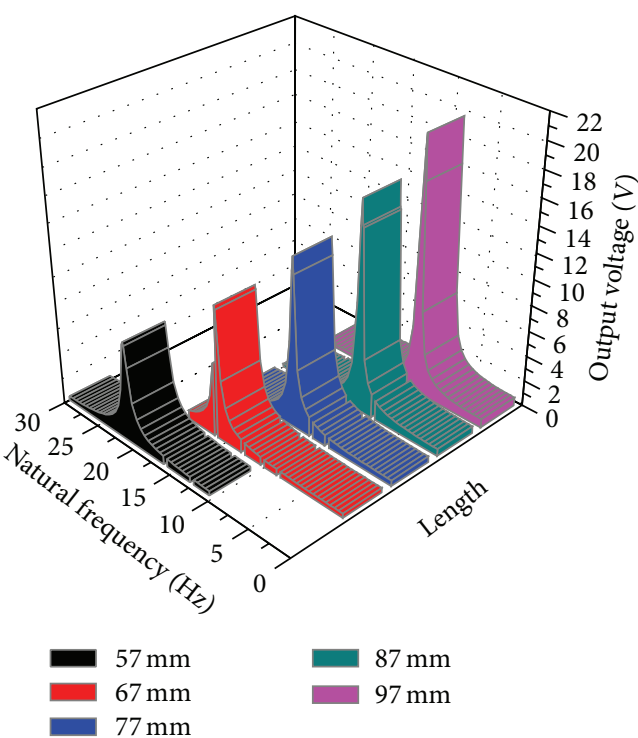

(b) Output voltage

Figure 7: Relationship between length and output of WPB.

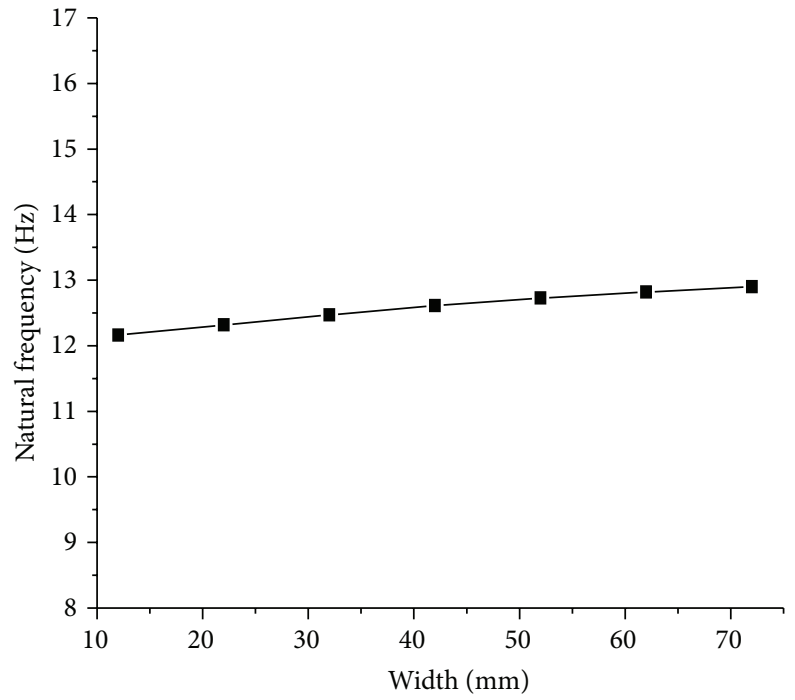

(a) Natural frequency

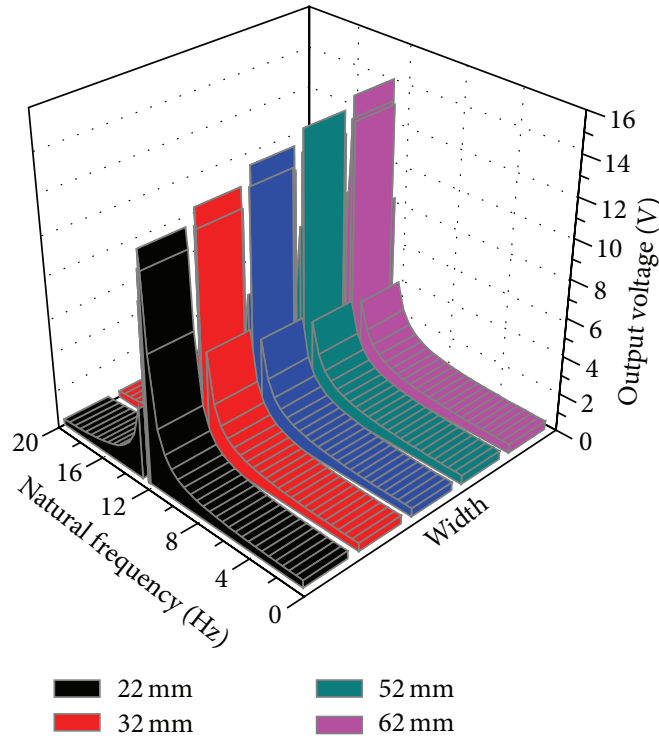

(b) Output voltage

FIGURE 8: Relationship between width and output of WPB.

generated by piezoelectric layers is always fluctuant, but it keeps rising in general and can easily reach the value simulated in ANSYS.

\section{Conclusions}

In this paper, a modal response theory model is built. The static analysis, modal analysis, and piezoelectric harmonic response analysis of WPB are given in detail by using ANSYS software. The structure stress and the displacement of WPB under wind load are discussed, as well as the relationship among the output voltage, natural frequency, and the size of WPB. An experiment of PWB is conducted. The main conclusions of this paper are as follows:

(1) According to the result of modal analysis, there is no irregular distortion under the first modal mode. 


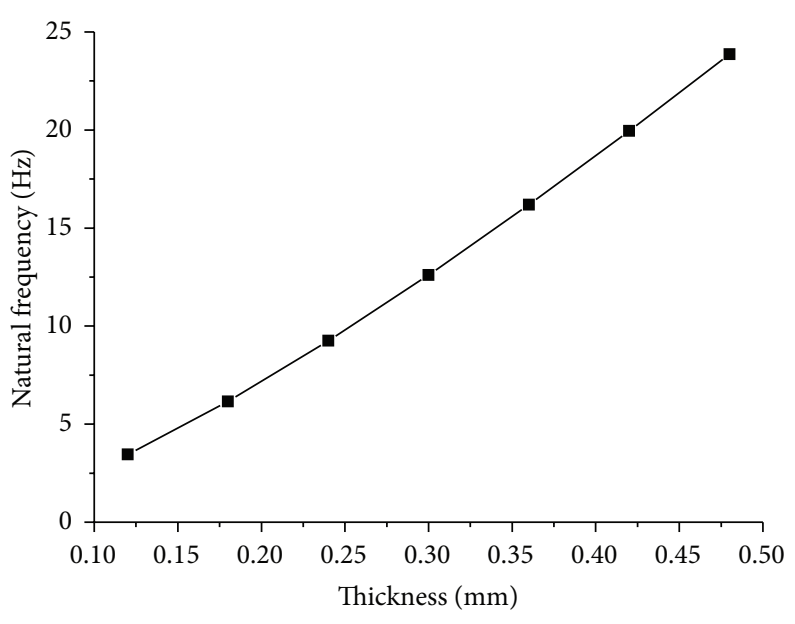

(a) Natural frequency

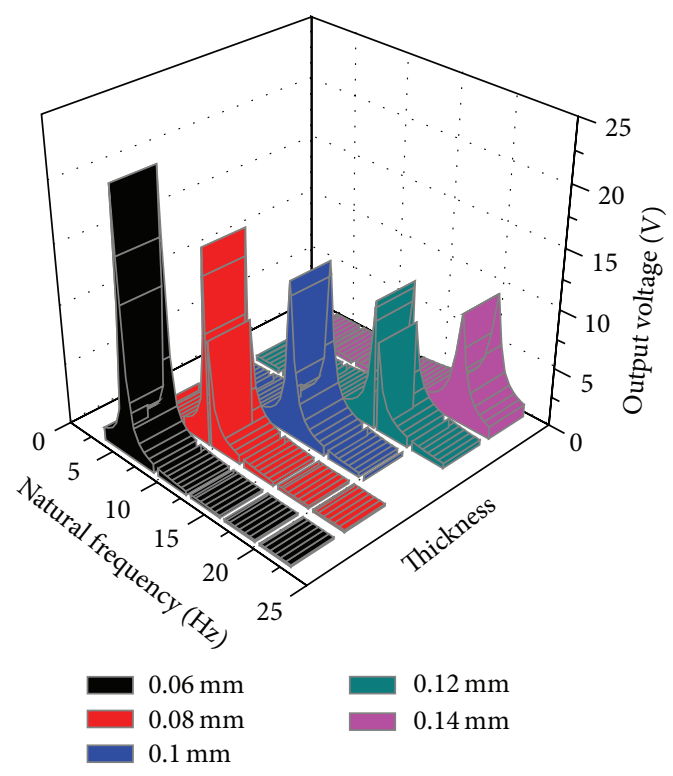

(b) Output voltage

FIGURE 9: Relationship thickness and output of WPB.

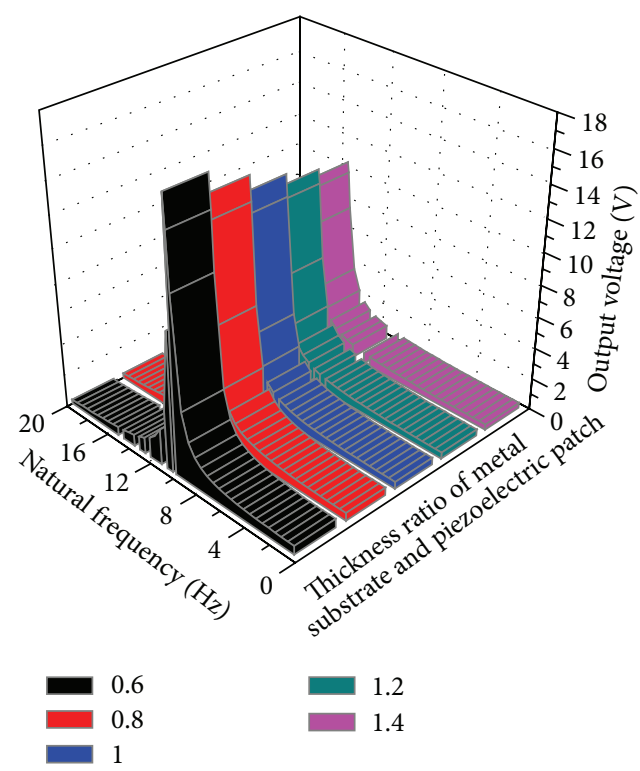

FIGURE 10: Relationship between the thickness ratio of metal substrate and piezoelectric patch and output voltage of WPB.

Therefore, the WPB has low resonant frequency and stable output under this condition.

(2) According to the result of harmonic response analysis, the WPB can achieve the maximum output voltage, which is $12.3 \mathrm{~V}$ under the resonant condition.

(3) The natural frequency decreases with the increase of the length of WPB and the free end mass. However, it will be increased when the width and thickness of WPB are increasing.
(4) The output voltage increases with the increase of the length and width, but the increasing amplitude is relatively smaller. In addition, the output voltage decreases with the increase of the thickness of WPB and the ratio of metal substrate to piezoelectric patches thickness. However, it will be changed little with the increase of the free end mass.

(5) The output voltage in the experiment can reach the value simulated in ANSYS. 


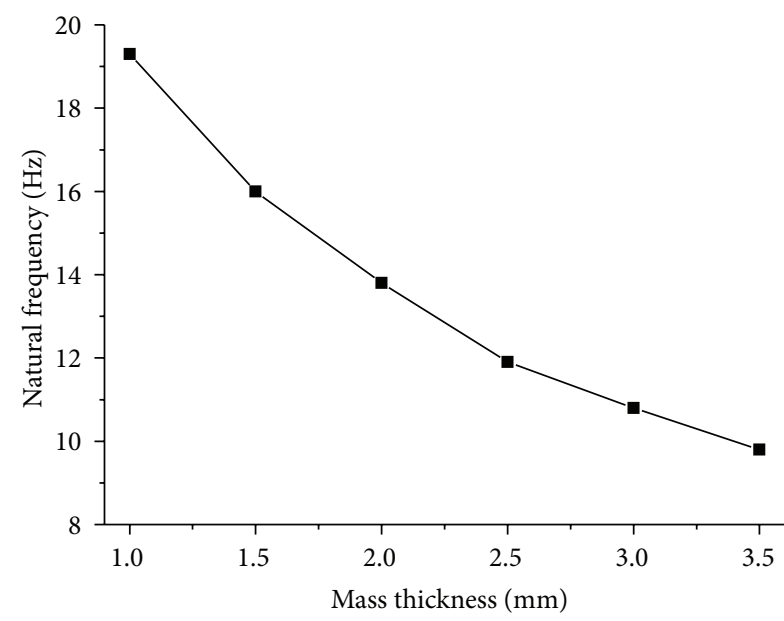

(a) Natural frequency

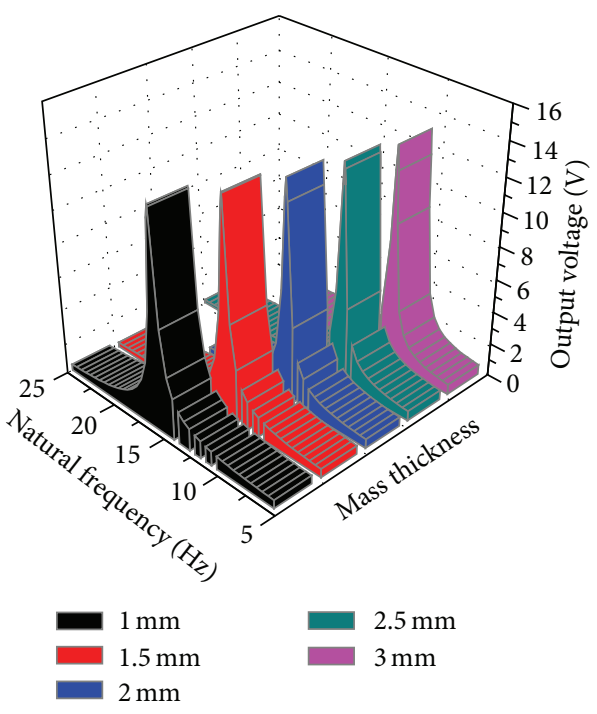

(b) Output voltage

FIGURE 11: Relationship between the end mass thickness and output of WPB.

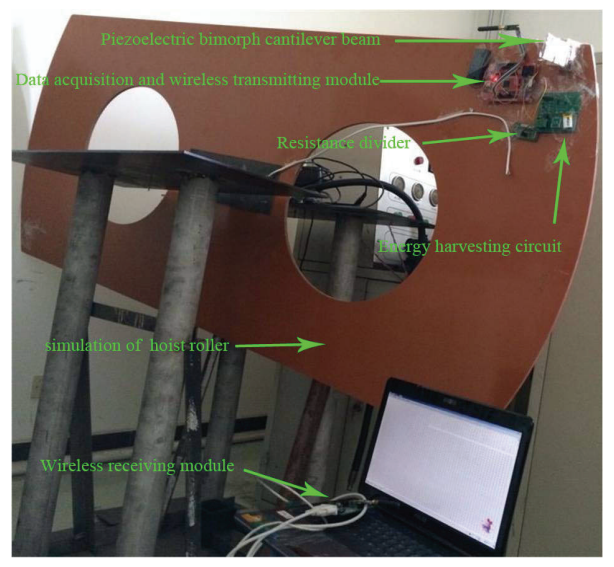

FIGURE 12: Experimental facilities of wind-induced piezoelectric energy harvesting device.

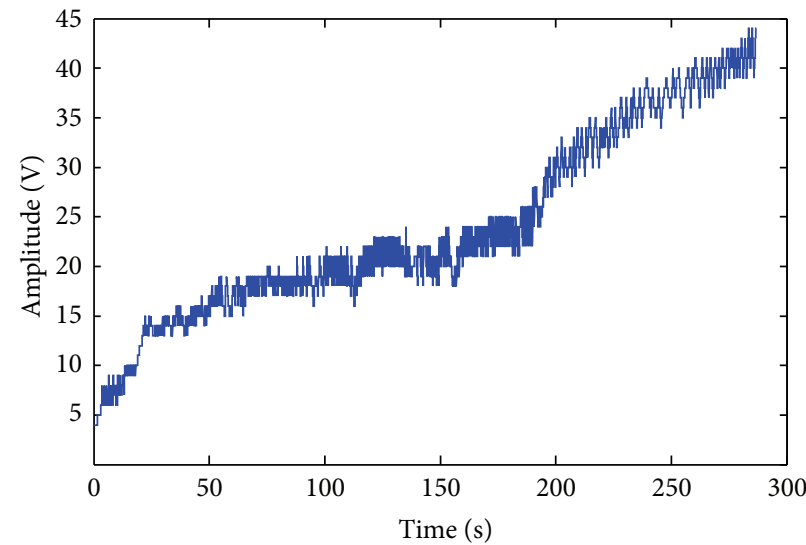

FIGURE 13: Output voltage amplitude of piezoelectric energy harvesting device. 


\section{Conflict of Interests}

The authors declare that there is no conflict of interests regarding the publication of this paper.

\section{Acknowledgments}

The material presented in this paper is based upon work supported by National Natural Science Foundation of China (no. 51275513), by the Fundamental Research Funds for the Central Universities (no. 2015QNA37), and by the Priority Academic Program Development of Jiangsu Higher Education Institutions.

\section{References}

[1] X. Gao, W.-H. Shih, and W. Y. Shih, "Flow energy harvesting using piezoelectric cantilevers with cylindrical extension," IEEE Transactions on Industrial Electronics, vol. 60, no. 3, pp. 11161118, 2013.

[2] S. Roundy, D. Steingart, L. Frechette et al., "Power sources for wireless sensor networks," in Wireless Sensor Networks, Lecture Notes in Computer Science, pp. 1-17, Springer, Berlin, Germany, 2004.

[3] D. Zhu, S. Beeby, J. Tudor, N. White, and N. Harris, "A novel miniature wind generator for wireless sensing applications," in Proceedings of the 9th IEEE Sensors Conference (SENSORS '10), pp. 1415-1418, November 2010.

[4] D. Ramasur and G. P. Hancke, "A wind energy harvester for low power wireless sensor networks," in Proceedings of the IEEE International Instrumentation and Measurement Technology Conference (I2MTC '12), pp. 2623-2627, May 2012.

[5] Y. K. Tan and S. K. Panda, "A novel piezoelectric based wind energy harvester for low-power autonomous wind speed sensor," in Proceedings of the 33rd Annual Conference of the IEEE Industrial Electronics Society (IECON '07), pp. 2175-2180, November 2007.

[6] J.-L. Wang, B.-H. Bao, F.-L. Wen, and Y.-Z. Gong, "Modeling and analysis of bimorph cantilever generators," Machinery Design \& Manufacture, no. 9, pp. 3-5, 2010.

[7] A. Khaligh, P. Zeng, and C. Zheng, "Kinetic energy harvesting using piezoelectric and electromagnetic technologies-state of the art," IEEE Transactions on Industrial Electronics, vol. 57, no. 3, pp. 850-860, 2010.

[8] S. Priya, C.-T. Chen, D. Fye, and J. Zahnd, "Piezoelectric windmill: a novel solution to remote sensing," Japanese Journal of Applied Physics, Part 2: Letters, vol. 44, no. 1-7, pp. L104-L107, 2005.

[9] X. Tang and L. Zuo, "Enhanced vibration energy harvesting using dual-mass systems," Journal of Sound and Vibration, vol. 330, no. 21, pp. 5199-5209, 2011.

[10] M. Arafa, W. Akl, A. Aldwani et al., "Experimental implementation of a cantilevered piezoelectric energy harvester with a dynamic magnifier," in Active and Passive Smart Structures and Integrated Systems 2011, vol. 7977 of Proceedings of SPIE, The International Society for Optical Engineering, April 2011.

[11] V. R. Challa, M. G. Prasad, Y. Shi, and F. T. Fisher, "A vibration energy harvesting device with bidirectional resonance frequency tunability," Smart Materials and Structures, vol. 17, no. 1, Article ID 015035, 2008.
[12] J. W. Xu, W. W. Shao, F. R. Kong, and Z. H. Feng, "Rightangle piezoelectric cantilever with improved energy harvesting efficiency," Applied Physics Letters, vol. 96, no. 15, Article ID 152904, pp. 15-29, 2010.

[13] H. A. Sodano, G. Park, and D. J. Inman, "Estimation of electric charge output for piezoelectric energy harvesting," Strain, vol. 40, no. 2, pp. 49-58, 2004.

[14] N. E. DuToit, B. L. Wardle, and S.-G. Kim, "Design consideration for MEMS-scale piezoelectric mechanical vibration energy harvests," Integrated Ferroelectrics, vol. 71, no. 1, pp. 121-160, 2005.

[15] M. G. Abd El-Mageed, M. Arafa, and M. Elaraby, "Simulation and experimental investigation of an energy harvester utilizing flow-induced vibration," in Proceedings of the ASME International Design Engineering Technical Conferences \& Computers and Information in Engineering Conference (IDETC/CIE '14), Paper No. DETC2014-34596, Buffalo, NY, USA, August 2014.

[16] T. Kiwata, M. Yamaguchi, A. Nakajima, T. Kono, and T. Ueno, "Flow-induced transverse vibration of a cantilevered prism for energy harvesting," in Proceeding of the ASME 2014 Pressure Vessels \& Piping Conference, Paper No. PVP2014-28939, Anaheim, Calif, USA, July 2014.

[17] A. Bibo, A. Abdelkefi, and M. F. Daqaq, "Modeling and characterization of a piezoelectric energy harvester under combined aerodynamic and base excitations," Journal of Vibration and Acoustics, vol. 137, pp. 3-6, 2015.

[18] Y. Zhi-Jie, The analysis of dynamic response of pipeline under vortex-induced force [M.S. thesis], Zhejiang University, 2010. 

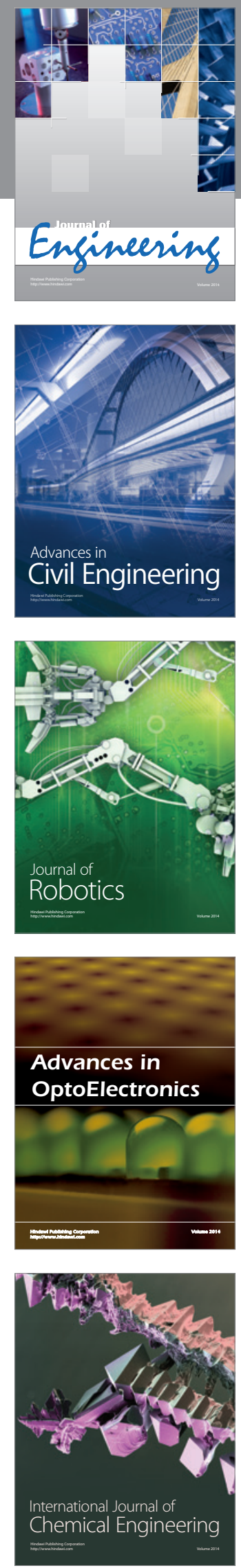

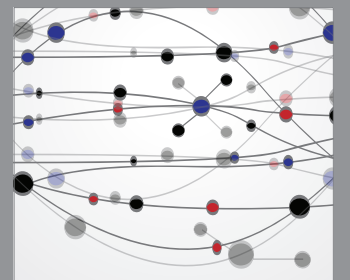

The Scientific World Journal
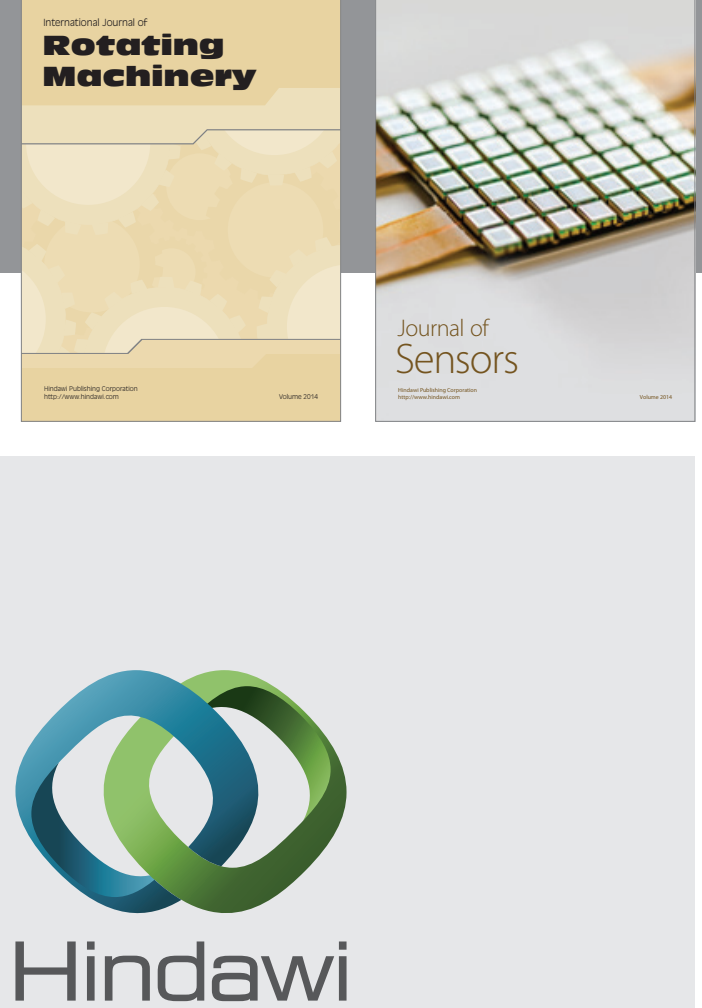

Submit your manuscripts at http://www.hindawi.com
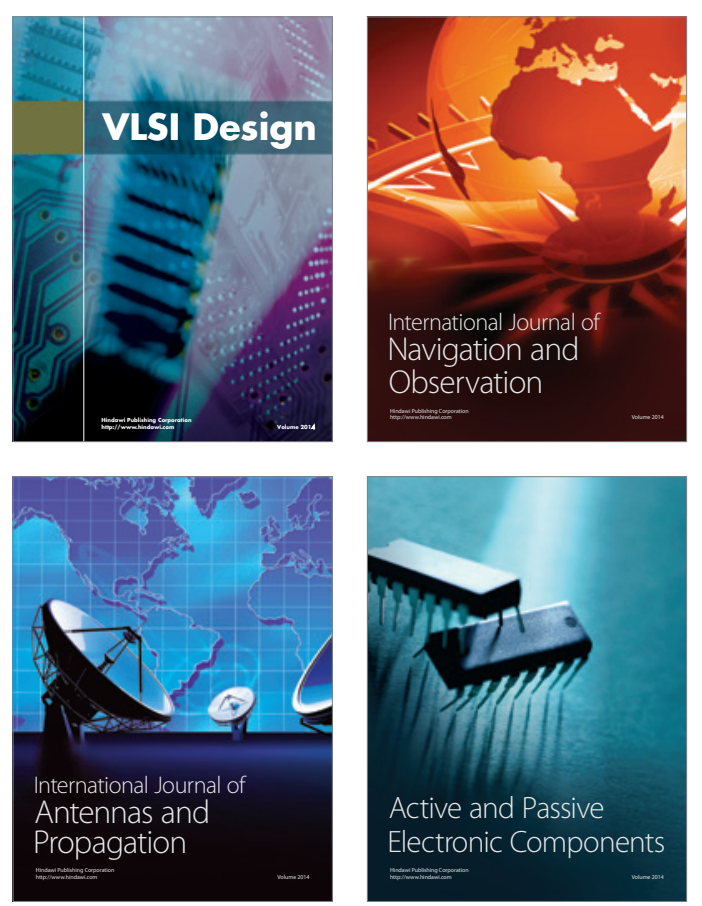
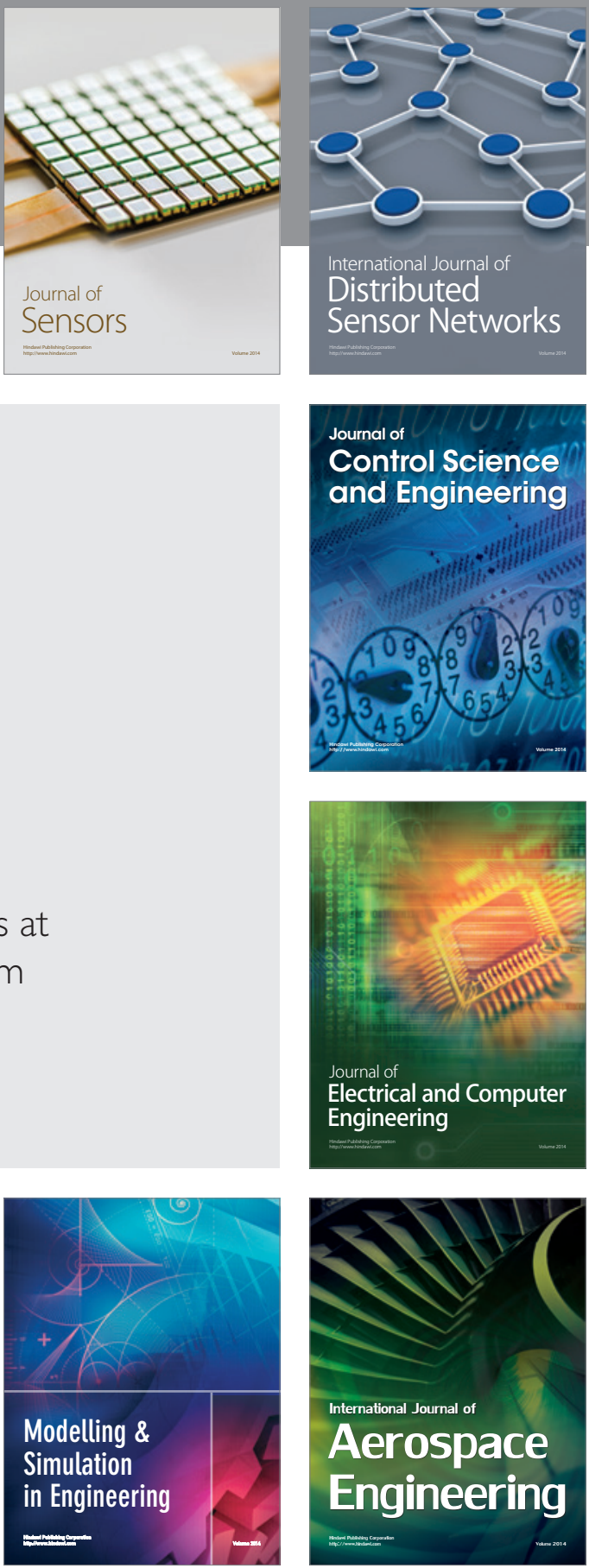

Journal of

Control Science

and Engineering
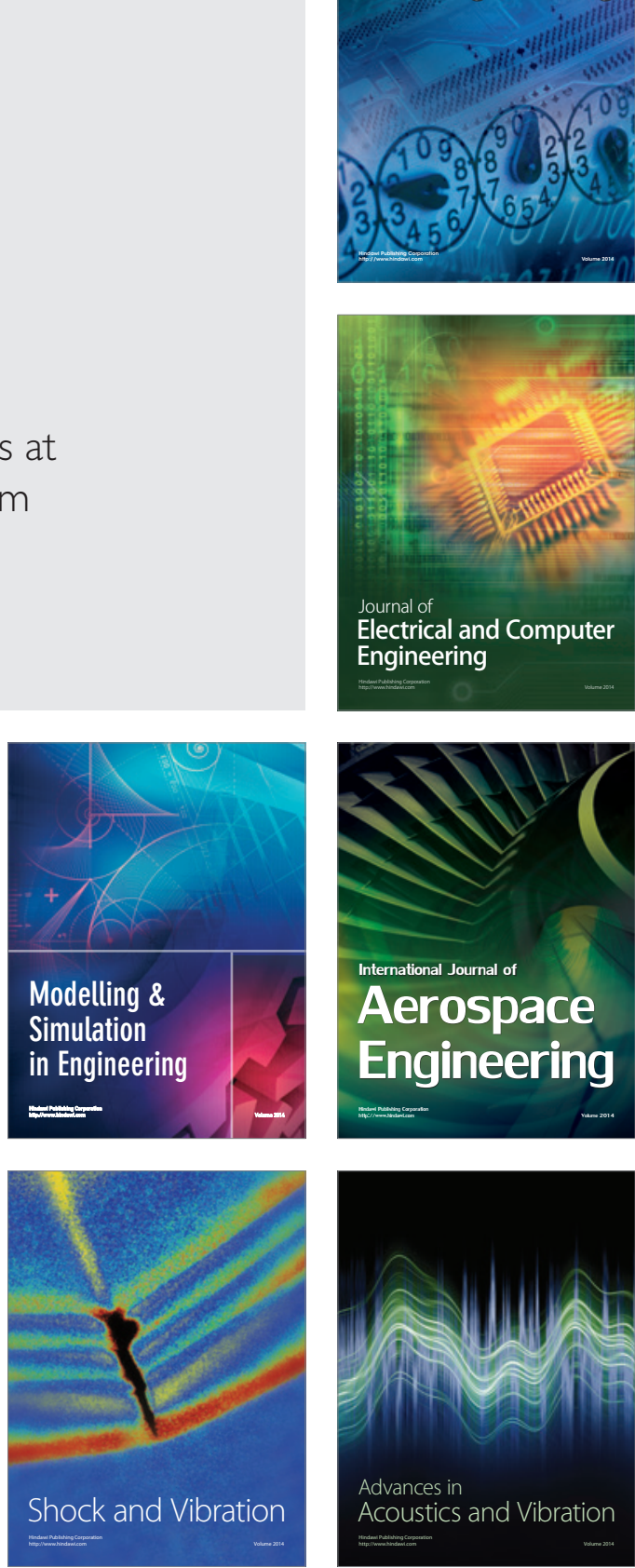